\title{
The radial dependence of temperature and iron abundance
}

\section{Galaxy clusters from $z=0.14$ to $z=0.89$}

\author{
S. Ehlert ${ }^{1,3}$ and M. P. Ulmer ${ }^{2,3}$ \\ 1 Max-Planck-Institut für Kernphysik, PO Box 103980, 69029 Heidelberg, Germany \\ e-mail: Steven.Ehlert@mpi-hd.mpg.de \\ 2 LAM, Pôle de l'Etoile Site de Château-Gombert, 38, rue Frédéric Joliot-Curie, 13388 Marseille Cedex 13, France \\ e-mail: m-ulmer2@northwestern.edu \\ 3 Department of Physics and Astronomy, Northwestern University, 2131 Sheridan Road, Evanston, IL 60208-2900, USA
}

Received 19 March 2008 / Accepted 3 June 2009

\begin{abstract}
Context. The origin and evolution of the intracluster medium (ICM) are still not fully understood. A better understanding is not only interesting in its own right, but it is also important for modeling hierarchical structure growth and for using cluster surveys to determine cosmological parameters.

Aims. To determine if there exists any evidence for evolution in the temperature or iron abundance gradients between $z \simeq 0.14$ and $z \simeq 0.89$, therefore elucidating the origin of energy and metal input to the ICM.

Methods. By using a sample of 35 observations of 31 clusters of galaxies found in the archival data of Chandra and XMM-Newton with redshifts between 0.14 and 0.89 , we derived the temperature and iron abundance radial profiles. To compare clusters with similar properties, the data were divided into comparable subsets.

Results. There is no substantial evidence to suggest that the iron abundance radial profiles in galaxy clusters evolve with redshift in any of the chosen subsets. Temperature radial profiles also do not appear to be changing with redshift once selection effects are taken into account.

Conclusions. The lack of evolution in the iron profiles is consistent with scenarios where the galaxies in clusters are stripped of their gas at higher redshifts. The temperature and iron abundance profiles also suggest that the primary source of heating in high redshift clusters is the gravitational infall of mass. These findings further emphasize the importance of modeling the local environment of clusters in cosmological studies and have important implications for studies that go to larger redshifts.
\end{abstract}

Key words. X-rays: galaxies: clusters

\section{Introduction}

Many dynamic processes occur in the high temperature, iron rich intracluster medium (ICM) of rich clusters of galaxies. Radiative cooling, supernova driven winds, and ram pressure stripping are just a few of the processes by which thermal energy and iron may be injected into the ICM. The theory of these processes is discussed in detail elsewhere (e.g., Sarazin 1988; Rosati et al. 2002; Arnaud 2005). In order to help resolve which processes most significantly contribute to the heating and iron enrichment within clusters, the location and evolution with redshift of both iron abundance and thermal energy can be used to provide significant clues.

Both Chandra and XMM-Newton have the angular resolution to make two-dimensional maps for nearby clusters such as Coma and Perseus (e.g., Fabian et al. 2006), but the statistical significance of the archival data is not generally sufficient for these types of measurements in distant clusters.

Many have already investigated how overall temperatures or iron abundances might vary with redshift, including Balestra et al. (2007), Bonamente et al. (2006), Tozzi et al. (2003), and Sanderson et al. (2006). So far very little evidence for the evolution of the ICM in galaxy clusters up to $z \simeq 1.0$ has been discovered (Matsumoto et al. 2000), but even subtle hints of changes with redshift could have a profound impact on the general understanding of the processes involved in the ICM. This current study spatially resolves the temperature and metal (iron) abundance to higher redshifts than previous works, as well as determines whether there exists evidence for changes in the temperature or iron abundance profile with redshift.

For higher redshift clusters, studies of the evolution of the radial temperature and metallicity profiles has been reported out to a redshift $\sim 0.3$ (Leccardi \& Molendi 2008b,a), and here the work is extended out to $\sim 0.9$. The goal is to search for evolutionary changes in the radial profiles. The results can be used to determine the relative significance of processes such as ram pressure stripping, Active Galactic Nucleus (AGN) activity, and galactic winds in clusters of galaxies. The end results can then be compared directly to simulations such as Domainko et al. (2006). Throughout this paper the concordance model of cosmology with $H_{0}=71 \mathrm{~km} \mathrm{~s}^{-1} \mathrm{Mpc}^{-1} \Lambda_{0}=0.73$ and $\Omega_{\mathrm{m}, 0}=0.27$ is assumed. All calculations of cosmic distances and times are based on Wright (2006).

\section{The data}

All data in this study were taken from the Chandra and XMM-Newton public archives, and are listed in Tables 1 and 2. 
Table 1. Basic information about Chandra data sets used in this study.

\begin{tabular}{ccccccc}
\hline \hline & \multicolumn{7}{c}{ Clusters } & analyzed from Chandra & \\
cluster name & Obs \# & RA & Dec & $z$ & $n_{\mathrm{H}}\left(10^{22} \mathrm{~cm}^{-2}\right)$ & exposure time (s) \\
\hline Abell 1413 & 1661 & 115518.20 & +232428.80 & 0.142 & 0.0219 & 9749 \\
Abell 2204 & 499 & 163247.00 & +053433.00 & 0.152 & 0.0567 & 11250 \\
Abell 665 & 3586 & 083045.20 & +655255.00 & 0.182 & 0.0431 & 28600 \\
RX J0439.0+0520 & 527 & 043902.20 & +052043.00 & 0.208 & 0.1070 & 10830 \\
Abell 773 & 533 & 091759.40 & +514223.00 & 0.217 & 0.0126 & 10500 \\
Abell 697 & 4217 & 084253.30 & +362012.00 & 0.282 & 0.0341 & 18880 \\
Abell 611 & 3194 & 080056.90 & +360326.00 & 0.288 & 0.0499 & 35990 \\
MS 1008.1-1224 & 926 & 101032.33 & +123932.18 & 0.306 & 0.0726 & 42960 \\
MS 2137.3-2353 & 928 & 214012.70 & -233927.00 & 0.313 & 0.0355 & 31990 \\
Abell 1995 & 906 & 145250.40 & +580248.00 & 0.319 & 0.0145 & 43830 \\
ZWCL 1358+6245 & 516 & 135950.60 & +623104.00 & 0.328 & 0.0193 & 45420 \\
MACS J2228.5+2036 & 3285 & 222834.40 & +203647.00 & 0.412 & 0.0429 & 19700 \\
MACS J2214.9-1359 & 3259 & 221457.30 & -140014.00 & 0.483 & 0.0328 & 17020 \\
MACS J1311.0-0310 & 3258 & 131101.70 & -031041.00 & 0.490 & 0.0188 & 15000 \\
MS 0015.9+1609 & 520 & 001833.86 & +162607.75 & 0.541 & 0.0407 & 66940 \\
MACS 1423.8+2404 & 4195 & 142347.80 & +240441.40 & 0.545 & 0.0238 & 113400 \\
MS 0451.6-0305 & 529 & 045410.90 & -030107.20 & 0.550 & 0.0500 & 16370 \\
MS 0451.6-0305 & 902 & 045410.90 & -030107.20 & 0.550 & 0.0500 & 41470 \\
MACS J2129.4-0741 & 3199 & 212926.20 & -074127.00 & 0.570 & 0.0484 & 17690 \\
MS 2053.7-0449 & 551 & 205622.40 & -043743.00 & 0.583 & 0.0462 & 43100 \\
MS 2053.7-0449 & 1667 & 205622.40 & -043743.00 & 0.583 & 0.0462 & 43900 \\
MACS J0647.7+7015 & 3196 & 064750.20 & +701455.00 & 0.584 & 0.0563 & 18850 \\
CL 1120+4318 & 5771 & 112007.60 & +431807.00 & 0.600 & 0.0208 & 19740 \\
MACS J0744.8+3927 & 3197 & 074453.00 & +392726.00 & 0.686 & 0.0568 & 19690 \\
MS 1137.5+6625 & 536 & 114023.30 & +660709.00 & 0.782 & 0.0121 & 119500 \\
RX J1716.9+6708 & 548 & 171652.30 & +670831.20 & 0.813 & 0.0372 & 50350 \\
CL J1226.9+3332 & 3180 & 122658.20 & +333248.00 & 0.890 & 0.0137 & 29470 \\
\hline
\end{tabular}

Most of these observations were chosen after being included in Bonamente et al. (2006), Tozzi et al. (2003) or Leccardi \& Molendi (2008b). All clusters listed here that are not found in these studies were found by searching the Chandra and $X M M-N e w t o n$ archives for galaxy cluster observations. The resulting sample was chosen to cover a large range of redshift with reasonable uniformity. This allowed for the sample to be conveniently divided into subsets which will be compared to one another, building on previous work at lower redshifts (De Grandi et al. 2004). The redshifts and coordinates of these clusters were provided by the NASA/IPAC Extragalactic Database (NED), and the hydrogen column densities are given by the $n_{\mathrm{H}}$ calculator provided on the HEASARC website. Investigations into the element abundances of galaxy clusters suggest that most of the elemental emission is dominated by iron, and that many other elements have abundance levels that do not change much with redshift (Baumgartner et al. 2005). This allowed for the use of iron abundance and metal abundance interchangeably, and although the model used in this work fit the abundance of all metals, the results of these fits will hereafter be called the iron abundance measurements.

\subsection{Chandra data processing}

All Chandra observations used herein were observed with the ACIS camera. All processing of Chandra data was carried out using CIAO 3.3 and all the contained packages including Sherpa and ChIPS and followed the general procedure found in Tozzi et al. (2003). For each data set the standard issue EVENTS LEVEL 1 file was reset to remove all corrections already implemented on the data set. The process acisrunbadpix was run on the reset file to detect all bad pixels. Next, the standard Level 1 events processing, acisprocessevents, was run on the reset file to create a new EVENTS LEVEL 1 file. Detection of very faint pixels was done whenever the data itself was taken in VFAINT mode. The new Level 1 file was then filtered to only include the standard event grades $0,2,3,4$, and 6 and also filtered further with the pipeline good time intervals to create a new EVENTS LEVEL 2 file. The new Level 2 file was processed further using the destreak routine and then using the analyzeltcrv routine as part of the ChIPS package to determine in greater detail the good time intervals. The bin time used for all data sets was $200 \mathrm{~s}$. The de-streaked file was then filtered again using these good time intervals. As a final step in processing, the image was then filtered only to include the energy range of $0.3-10 \mathrm{keV}$. Filtering on the energy improves the signal to noise ratio of the image. The resulting event list was then used in all subsequent imaging and spectral analysis. There are 25 clusters observed using 27 Chandra observations, with redshifts ranging from 0.142 to 0.890 . All of the cluster data sets, along with their Equatorial coordinates, redshifts, galactic hydrogen column densities, and net exposure times after all processing are listed in Table 1.

\subsection{XMM-Newton data processing}

All XMM-Newton data analyzed in this project used all three EPIC cameras: MOS1, MOS2, and the PN camera. The software used was SAS version 7.0, the standard XMM-Newton processing software. All data sets were processed using the images script available on the XMM-Newton website. The images script first runs the fundamental SAS algorithms cifbuild, odfingest, epchain, and emchain. It then processes the standard event list further to improve signal-to-noise. In order to minimize the significance of flare events, the images script allows for the user to input desired Good-Time-Interval (GTI) for each data set. In this case the GTI intervals were chosen as time windows where 
Table 2. Basic information about XMM-Newton data sets used in analysis.

\begin{tabular}{ccccccc}
\hline \hline & \multicolumn{6}{c}{ Clusters analyzed from XMM-Newton } \\
cluster name & Obs \# & RA & Dec & $z$ & $n_{\mathrm{H}}\left(10^{22} \mathrm{~cm}^{-2}\right)$ & exposure time $(\mathrm{s})$ \\
\hline Abell 1763 & 0084230901 & 133517.20 & +405958.00 & 0.223 & 0.0082 & $19500 / 19500 / 9197$ \\
Abell 2390 & 0111270101 & 215334.60 & +174011.00 & 0.228 & 0.0619 & $13900 / 13900 / 9445$ \\
Abell 1835 & 0147330201 & 140102.00 & +025132.00 & 0.253 & 0.0204 & $78120 / 79450 / 32060$ \\
RX J0256.5+0006 & 0056020301 & 025629.51 & +000528.70 & 0.360 & 0.0650 & $19190 / 19170 / 7914$ \\
RX J0318.2-0301 & 0056022201 & 031828.76 & -030046.70 & 0.370 & 0.0505 & $20270 / 20340 / 11570$ \\
RX J0426.1+1655 & 0056020401 & 042604.20 & +165548.50 & 0.380 & 0.1910 & $18820 / 18820 / 8337$ \\
RX J1120.1+4318 & 0107860201 & 112000.91 & +431815.10 & 0.600 & 0.0203 & $22140 / 22150 / 16240$ \\
CL J1226.9+3332 & 0200340101 & 122658.00 & +333254.00 & 0.890 & 0.0138 & $77750 / 77780 / 60660$ \\
\hline
\end{tabular}

Note: the exposure times for the XMM-Newton data sets are given in the following order: MOS1/MOS2/PN.

the rate was less than 35 counts per $100 \mathrm{~s}$ for the MOS cameras, and 40 counts per $100 \mathrm{~s}$ for the PN camera. After GTI filtering the images script then cleans for bad pixels based on a separate input script, with default bad pixels provided by the $X M M-N e w t o n$ data center. The standard bad pixel table provided by the images script was used here. Although the images script was originally designed to create high quality images from all three cameras, the resulting event lists are still useful for spectral studies. Unlike the case of the Chandra data sets, the event lists used for XMM-Newton analysis have not been subject to any energy filtering before determining radial profiles. However, all spectra for both instruments are restricted to the energy range from $0.5-8.0 \mathrm{keV}$. As a final restriction, the PATTERNS on the events were limited to single, double, triple, and quadruple events for the MOS cameras (PATTERN $\leq 12$ ) and limited to single and double events for the PN camera (PATTERN $\leq 4$ ). Eight XMM-Newton data sets were considered in this study. They are listed along with Equatorial coordinates, redshifts, hydrogen column density, and net exposure times after all processing in Table 2.

\subsection{Scale lengths: the core and counts radius}

In order to average clusters together in a meaningful way, a scale length was needed. Two scale lengths were calculated for each cluster and used to divide the cluster into four annular regions: the first scale length was calculated by fitting the net intensity to a one-dimensional beta fit and the second by dividing the cluster into four regions with roughly equal numbers of net counts. The outer radii in these regions are labeled $r_{1}, r_{2}, r_{3}$, and $r_{4}$ in Table 3. These radial profiles were determined using either the surface brightness (core) or the net counts (counts), and the background region for these calculations was always an annular region at least $5^{\prime \prime}$ wide. This annular region was usually sufficiently small to minimize contamination from line-of-sight point sources while also containing enough counts $(\simeq 100-1000)$ to be statistically significant $(\sim 3-10 \%)$. In some cases, the background region needed to be larger to reach this level of significance, but a region with a significant number of counts $(\geq 100)$ was always used. The profiles are not strongly sensitive to the number of counts used in the background regions, which were chosen specifically to be radially symmetric. For each cluster, both radii have been calculated using annular regions 2 .'5 wide to determine the intensity profile across the cluster. Both scale radii were then used to determine regions for extracting spectra and measuring the average temperature and iron abundance profiles. The only profiles and measurements shown in the text will use the counts radius, the reasons for which will be discussed in Sect. 2.3.3. The measured core radius and counts regions are found in Table 3 .

\subsubsection{The core radius}

The basis for using a core radius length scale is that cluster dynamics and the evolution of the ICM could be inter-related (e.g., Rosati et al. 2002), so a one-dimensional beta fit was performed on each cluster to determine the core radius scale. The centers were chosen by eye, and consistent with all attempts to determine the center of emission analytically. The error for choosing this center was always a factor of 10 smaller than the calculated core radius. The radial profile of surface brightness was calculated using the the CIAO command dmextract, and the net counts annuli described in Sect. 2.3, and it was fit to the Sherpa betald model. The betald mode fits the data to a function of the form

$I(x)=A \times\left[1+\left(\frac{x-x_{0}}{r_{\mathrm{c}}}\right)^{2}\right]^{-3 \beta+1 / 2}$

where $r_{\mathrm{c}}$ is the core radius. The parameter $\beta$ is a measure of the ratio of kinetic energy in the galaxies moving in the cluster to the thermal energy of the cluster (e.g., Rosati et al. 2002). The radial fits are described by the two parameters $r_{\mathrm{c}}$ and $\beta$. The offset from zero $\left(x_{0}\right)$ was always fixed at zero, and the proportionality constant normalizes the fit to the particular data relating it to the central density of the cluster. Radial profiles are listed in Table 3, with the first eight observations being from XMM-Newton. More elaborate fitting profiles like a double- $\beta$ fit (Bonamente et al. 2006) could have been used in lieu of a single- $\beta$ fit, but a single$\beta$ fit is usually all that statistics require (e.g., Ettori et al. 2004a). For clusters with high surface brightness in their center, however, a more appropriate fit may be the more elaborate model used by Kotov \& Vikhlinin (2005).

\subsubsection{The counts radius}

Upon examination of the one-dimensional beta fits and their reduced $\chi^{2}$ statistics, it appeared as though the beta model fits derived from Sherpa were not statistically robust. The reduced $\chi^{2}$ for these fits was often above 2, and although others have used similar results (Sakelliou \& Ponman 2004), it was judged necessary to find a second method used to independently verify the profiles. Therefore, a second set of temperature and iron profiles were calculated with the same set of data. The second method used was to divide the cluster into radial regions not by a constant length scale, but instead by giving each region a constant number of counts. The furthest extent of spectral extraction was 
Table 3. Radial profile parameters for all clusters. The XMM-Newton clusters are listed first.

\begin{tabular}{|c|c|c|c|c|c|c|c|c|c|}
\hline \multicolumn{10}{|c|}{ Radial profile parameters* } \\
\hline cluster name & RA (Center) & Dec (Center) & $\beta$ & core radius & $r_{1}$ & $r_{2}$ & $r_{3}$ & $r_{4}$ & $r_{200}$ \\
\hline Abell 1763 & $13: 35: 18$ & $+40: 59: 59$ & $0.95 \pm 0.03$ & $77.6_{-26}^{+2.4}$ & 32.5 & 22.5 & 30 & 37.5 & 717.0 \\
\hline Abell 2390 & $21: 53: 37$ & $17: 41: 40$ & $0.572 \pm 0.004$ & $23.4 \pm 0.4$ & 22.5 & 22.5 & 30 & 70 & 729.1 \\
\hline Abell 1835 & 14:01:02 & $02: 52: 40$ & $0.635 \pm 0.002$ & $18.88 \pm 0.1$ & 12.5 & 12.5 & 17.5 & 57.5 & 549.8 \\
\hline RX J0256.5+0006 & $02: 56: 34$ & $+00: 06: 25$ & $0.74_{-0.01}^{+0.30}$ & $42.7_{-0.7}^{+20.1}$ & 22.5 & 15 & 17.5 & 20 & 249.3 \\
\hline RX J0318.2-0301 & $03: 18: 34$ & $-03: 02: 59$ & $0.81_{-0.02}^{-0.01}$ & $42.3_{-1.3}^{+0.7}$ & 20 & 15 & 17.5 & 22.5 & 253.8 \\
\hline RXJ0426.1+1655 & 04:26:08 & $+16: 55: 14$ & $0.61 \pm 0.02$ & $15.9 \pm 1.3$ & 12.5 & 7.5 & 10 & 25 & 234.9 \\
\hline RXJ1120.1+4318 & 11:20:08 & $43: 18: 05$ & $0.93 \pm 0.07$ & $40.2 \pm 2.9$ & 17.5 & 10 & 15 & 20 & 149.2 \\
\hline CL J1226.9+3332 & $12: 26: 58$ & $3: 32: 45$ & $0.85 \pm 0.03$ & $23.6 \pm 0.9$ & 12.5 & 7.5 & 10 & 22.5 & 146.8 \\
\hline Abell 1413 & & $3: 24: 13$ & $0.85 \pm 0$ & $45.3 \pm 3.3$ & 25 & 17.5 & 25 & 57.5 & 764.4 \\
\hline Abell 2204 & $32: 47$ & $+05: 34: 27$ & $0.551 \pm 0.003$ & $7.5 \pm 0.2$ & 10 & 12.5 & 22.5 & 80 & 558.4 \\
\hline Abell 665 & :30:59 & $+65: 50: 12$ & $0.63 \pm 0.02$ & $36.6 \pm 2.2$ & 27.5 & 20 & 25 & 50 & 893.3 \\
\hline RX J0439.0+0520 & $04: 3$ & $+05: 20: 46$ & $0.58 \pm 0.02$ & $10.3 \pm 1.4$ & 10 & 12.5 & 20 & 50 & 369.4 \\
\hline Abell 773 & & $1: 43: 47$ & $1.51 \pm 0$ & $93.2 \pm 11.3$ & 27.5 & 15 & 20 & 45 & 743.7 \\
\hline Abell 611 & & $6: 03: 27$ & $0.85 \pm 0.04$ & $28.8 \pm 1.4$ & 15 & 12.5 & 17.5 & 55 & 360.5 \\
\hline Abel & & $+36: 20: 12$ & $0.98 \pm 0.06$ & $67.6 \pm 3.8$ & 30 & 20 & 25 & 67.5 & 448.1 \\
\hline MS 1008.1-1224 & $10: 10: 33$ & $-12: 39: 59$ & $0.69 \pm 0.04$ & $38.9 \pm 3.6$ & 25 & 20 & 25 & 55 & 328.2 \\
\hline MS 2137.3 & & $-23: 39: 43$ & $0.70 \pm$ & $9.8 \pm 0.3$ & 7. & 7. & 15 & 70 & 270.5 \\
\hline Abe & & $+58: 02: 48$ & $1.43 \pm$ & $69.1 \pm 5.5$ & 25 & 15 & 20 & 50 & 382.0 \\
\hline ZWCL $1358+6245$ & 59:51 & $+62: 31: 04$ & $0.57 \pm 0.01$ & $4 \pm 0.8$ & 12.5 & 15 & 20 & 42.5 & 289.5 \\
\hline MACS J2228.5+2036 & $22: 28: 34$ & $+20: 37: 23$ & $1.47 \pm 0.29$ & $50.5 \pm 7.1$ & 15 & 10 & 12.5 & 27.5 & 278.5 \\
\hline MACS J2214.9-1359 & $22: 14: 58$ & $-14: 00: 15$ & $1.00 \pm 0.13$ & $36.6 \pm 5.1$ & 15 & 12.5 & 17.5 & 35 & 244.2 \\
\hline MACS J1311.0-0310 & 1 & $-03: 10: 31$ & $1.13 \pm 0.20$ & $23.7 \pm 4.4$ & 10 & 10 & 10 & 37.5 & 201.7 \\
\hline MS 00 & 33 & $+16: 26: 07$ & $1.08 \pm 0.09$ & $54.2 \pm 4.0$ & 22.5 & 15 & 20 & 57.5 & 221.0 \\
\hline MACS $1423.8+2404^{\dagger}$ & $14: 23: 49$ & $+24: 04: 35$ & $0.62 \pm 0.01$ & $6.6 \pm 0.3$ & 5 & 7.5 & 17.5 & 47.5 & 162.5 \\
\hline MS 0451.6-0305(\#529) & $04: 54: 12$ & $-03: 00: 58$ & $1.18 \pm 0.19$ & $51.7 \pm 7.5$ & 17.5 & 12.5 & 15 & 30 & 208.2 \\
\hline MS 0451.6-0305(\#902) & 11 & $-03: 00: 48$ & $0.83 \pm 0.03$ & $36.1 \pm 1.5$ & 20 & 15 & 20 & 60 & 224.6 \\
\hline MACS J2129.4-0741 & $21: 29: 27$ & $-07: 41: 26$ & $0.66 \pm 0.04$ & $18.6 \pm 2.2$ & 12.5 & 10 & 15 & 37.5 & 185.2 \\
\hline MS 2053.7-0449(both) & $20: 56: 22$ & $-04: 37: 47$ & $0.66 \pm 0.05$ & $18.2 \pm 2.1$ & 12.5 & 10 & 12.5 & 32.5 & 208.6 \\
\hline MACS J0647.7+7015 & $06: 47: 50$ & $+70: 14: 55$ & $0.91 \pm 0.08$ & $29.8 \pm 3.1$ & 15 & 10 & 15 & 37.5 & 233.8 \\
\hline RX J1120.1+4318 & $11: 20: 06$ & $+43: 18: 06$ & $1.17 \pm 0.31$ & $42.0 \pm 9.7$ & 15 & 10 & 12.5 & 22.5 & 141.3 \\
\hline MACS J0744.8+3927 & $07: 44: 52$ & $+39: 27: 29$ & $0.56 \pm 0.03$ & $8.0 \pm 1.4$ & 7.5 & 7.5 & 12.5 & 35 & 143.0 \\
\hline MS $1137.5+6625$ & $11: 40: 23$ & $+66: 08: 20$ & $0.86 \pm 0.07$ & $18.8 \pm 2.0$ & 10 & 7.5 & 10 & 27.5 & 133.1 \\
\hline RX J1716.9+6708 & $17: 16: 49$ & $+67: 08: 27$ & $0.89 \pm 0.19$ & $24.4 \pm 5.8$ & 12.5 & 10 & 12.5 & 37.5 & 124.4 \\
\hline CL J1226.9+3332 & $12: 26: 58$ & $+33: 32: 48$ & $1.29 \pm 0.30$ & $27.6 \pm 5.5$ & 10 & 7.5 & 10 & 27.5 & 141.8 \\
\hline
\end{tabular}

* All measurements of radial regions are given in arcseconds. ${ }^{\dagger}$ Denotes the two clusters with extremely small core radii. See Sect. 2.3.3.

also determined by the net counts in a 2.5 wide region. If the net counts next the 2 ". 5 wide region out ever dropped below 20 for Chandra or 100 for XMM-Newton data, then this defined the edge of the cluster due to their low signal-to-noise ratio of the regions beyond this point. The four calculated counts regions for each cluster are listed in Table 3. Dividing the cluster in this manner also allows for all of the spectra for one cluster to be comparably significant instead of having a wide disparity in spectral quality. The spectra were extracted in exactly the same manner as the core radius spectra, which will be described in detail in Sect. 2.5. This process of sub-dividing the cluster will be called the counts profile or counts radius, hereafter. They will also be listed as $r_{1}, r_{2}, r_{3}$, and $r_{4}$ as an abbreviation in the tables, particularly in Table 3

\subsubsection{Differences between the two profiles}

The radial profiles in each cluster are compared in two ways in Tables 3 and 4 . Table 3 shows the extents of each individual counts region as well as the calculated core radius and virial radius $r_{200}$ in arcseconds. Table 4 shows the average core and counts regions in terms of kpc. It was found that there were insignificant differences between the two radii on average, except for the very outer region. The outermost counts regions are usually much wider than $2 r_{\mathrm{c}}$ (see the averages given in Table 4). Therefore, the average temperature and iron abundance measurements should be strongly correlated between the core and counts radius, with differences only being expected in the outermost regions. Also included in Table 3 is the calculated virial radius in arcseconds. The virial radius is calculated as found in Jones et al. (2003), divided by 1.4 to take into account the difference between cosmologies $\left(H_{0}=50 \mathrm{~km} \mathrm{~s}^{-1} \mathrm{Mpc}^{-1}\right.$ in Jones et al. (2003) while $H_{0}=71 \mathrm{~km} \mathrm{~s}^{-1} \mathrm{Mpc}^{-1}$ here). The formula itself is given as

$r_{200}=2.779 \times\left(\frac{T}{10 \mathrm{keV}}\right)^{0.5} \times(1+z)^{-1.5} h_{71}^{-1} \mathrm{Mpc}$

and the temperature used was the overall temperature measured by the procedure described in Sect. 2.5. This allows for a direct comparison with other work that uses the virial radius. Typically 
Table 4. Average outer extent of the core and counts radius regions in $\mathrm{kpc}$.

\begin{tabular}{ccccc}
\hline \hline \multicolumn{5}{c}{ Outer extent of region in kpc } \\
radius & region \#1 & region \#2 & region \#3 & region \#4 \\
\hline Core & 87 & 173 & 260 & 347 \\
Counts & 84 & 177 & 314 & 607 \\
\hline
\end{tabular}

a counts region bin corresponds to about $0.1 r_{\text {virial }}$, thus the profiles discussed here extend out on average to about $0.4 r_{\text {virial }}$. Although the core and counts radius were both used as scale radii for temperature and iron abundance profiles, the measurements for the core radius profile have been omitted from this text. The core radius results have been omitted because of their weaker statistics as well as the presence of two very small core radius measurements, noted in Table 4 . The one-dimensional $\beta$-fit also suffers from bias in the presence of cool core clusters with high densities in the center. The radial profiles produced by the two different methods are very strongly correlated, and therefore the calculated results were not sensitive to this choice. Using the counts radius allows for more robust statistics and systematics.

\subsection{Excluded data}

Each cluster had to satisfy two general conditions before included in this study. Each cluster first needed to have a sufficient number of counts to measure a temperature that was significantly $(>2 \sigma)$ above zero. All of the clusters in this sample have more than an average of 1000 counts per detector (3000 for XMM-Newton observations, 1000 for Chandra observations) within two core radii, and the minimum luminosity of all clusters from zero to two core radii is $1 \times 10^{44} \mathrm{erg} \mathrm{s}^{-1}$. The second condition was morphological in nature, as it was necessary to have a reasonable $\beta$-fit to the data. Any observations that showed obvious visual signs of recent large merger activity were immediately excluded. Beyond that, several sets were excluded based on the values derived from the $\beta$-fit. In these cases the best fit core radius was larger than the furthest extent of the radial profile. These sets often had $\beta$ values that were unrealistically large as well, usually well above $\beta=2$. Since the fit was unreliable in both parameters, these clusters were not considered in this sample. Table 6 lists all the observations investigated, but not included, in this study and why they were not considered.

\subsubsection{The unusual case of $R X J 1347.5-1145$}

The very luminous cluster RX J1347.5-1145 was also investigated using two observations: Chandra observation \#3592 (Allen et al. 2002) and XMM-Newton observation \#0112960101 (Gitti \& Schindler 2005). Although it was sufficiently luminous and symmetric to be included here; the temperatures measured between these two observations did not agree within $3 \sigma$. After this work began, Ota et al. (2008) found a hot bubble in the southeast region of this cluster. Thus, it was judged that this cluster is not relaxed enough to be considered in this study and was also excluded.

\subsection{The spectra}

Spectra for each data set were extracted with routines in CIAO for Chandra or SAS for XMM-Newton. If the data set was from Chandra then the spectra were extracted using the specextract routine. If the data set was from XMM-Newton, then the OGIP
Spectral Products routine found in the Graphical Interface of SAS after running XMMSelect was used. Spectra were taken of all four annular regions in both scale lengths. When determining the average cluster temperature and iron abundance a circular region from $0-2 r_{\mathrm{c}}$ region was used. Background regions were always circles with radii comparable or larger than two core radii outside of the detectable emission and always on the same chip as the cluster image. The background regions remained consistent within each data set. These background regions are specifically chosen to contain as many counts as possible without including sources. Since radial symmetry is not a major concern with the spectral background, while the total number of counts is, this region is not the same background region used to determine the radial profiles. The software used to do the spectral fitting was XSPEC 12.4.0, and the MEKAL model in XSPEC was always used along with the TBABS galactic absorption model. Since the spectra often had low numbers of counts, the modified Cash statistic was always used to determine the best-fit temperature and iron abundance simultaneously. The modified Cash statistic is ideal for fitting spectra with a low number of counts in each bin (Nousek \& Shue 1989) and also allows for the use of a local background spectrum instead of fitting the background to a model. The only remaining free parameter in the fit was the normalization. The other parameters of the model were fixed: the redshifts were frozen at the values given by the NASA/IPAC Extragalactic Database (NED) while the hydrogen column densities were frozen to the values given by the $n_{\mathrm{H}}$ calculator on the HEASARC website. The solar abundance values used were those from Anders \& Grevesse (1989). For the XMM-Newton spectra, all three instruments were fit simultaneously to the same spectral model to ensure the best use of the available statistics. The measured temperature and iron abundance profiles for each cluster are found in Table 6. For the four clusters in this study with multiple observations, the temperature and iron abundance were determined by fitting all of the observations simultaneously.

\section{Selection effects}

In any population study, it is important to take into account selection effects within the sample itself. Two selection effects are important in this study: overall temperature and cooling time. These selection effects combined with the separation by redshift lead to eight different subsets of the sample which were then compared to one another. In Table 7, 8 subsets defined by their temperatures and cooling time are listed along with the number of clusters in each subset.

\subsection{Temperature and luminosity}

The average temperature of this sample of clusters follows a trend that needs to be taken into consideration. There is an inherent expectation that clusters at higher redshift should exhibit higher temperatures due to the $L_{\mathrm{x}}-T$ relationship (e.g., Ota et al. 2006; Pacaud et al. 2007; Ettori et al. 2002, and references therein). This is because more distant (higher redshift) clusters need to be more intrinsically luminous to be detected than low luminosity clusters. The X-ray bolometric luminosity is proportional to temperature as $L_{\mathrm{x}, \text { bol }} \propto T^{2.5}$ (e.g., Ota et al. 2006). Thus on average, the observed clusters at higher redshift should tend to higher average temperatures. It was necessary to take into account this selection effect when searching for variations with redshift. Based on the available sample, the boundary between high and low temperature clusters was set to $6.8 \mathrm{keV}$. 
Table 5. Observations investigated and not included in this study, along with the reasons they were excluded.

\begin{tabular}{cccc}
\hline \hline & \multicolumn{2}{c}{ Clusters not analyzed } & \\
cluster name & observatory & observation ID \# & reason for exclusion \\
\hline Abell 2163 & Chandra & 1653 & $\operatorname{Bad} \beta$-fit \\
Abell 2218 & Chandra & 1454 & $\operatorname{Bad} \beta$-fit \\
RX J122+4918 & Chandra & 1661 & $\operatorname{Bad} \beta$-fit \\
MACS J1149.5+2223 & Chandra & 1656 & $\operatorname{Bad} \beta$-fit \\
Abell 68 & Chandra & 3250 & $\operatorname{Bad} \beta$-fit \\
RCS J0439-2904 & Chandra & 3577 & Not enough counts \\
Abell 370 & Chandra & 515 & Bad $\beta$-fit \\
RX J1347.5-1145 & Chandra & 3592 & See Sect. 2.4.1 \\
RX J1200.8-0328 & XMM-Newton & 0056020701 & Not enough counts \\
WARP J0152.7-1357 & XMM-Newton & 0109540101 & Merger \\
RX J1334.3+5030 & XMM-Newton & 0111160101 & Merger \\
Sharc-2 & XMM-Newton & 0111160201 & Not enough counts \\
RX J1354.3-0222 & XMM-Newton & 0112250101 & Not enough counts \\
RX J1347.5-1145 & XMM-Newton & 0112960101 & See Sect. 2.4.1 \\
MS 1208.71+3928 & XMM-Newton & 0112190201 & Not enough counts \\
\hline
\end{tabular}

Table 6. Temperature and iron abundance measurements for all clusters by counts region.

\begin{tabular}{|c|c|c|c|c|c|c|c|c|c|}
\hline \multicolumn{2}{|l|}{ cluster information } & \multicolumn{5}{|c|}{$\begin{array}{l}\text { Temperatures and Iron Abundance Measurements } \\
\text { temperatures in } \mathrm{keV}\end{array}$} & \multicolumn{2}{|c|}{ iron abundances in $Z_{\odot}$} & \multirow[b]{2}{*}{ region \#4 } \\
\hline cluster name & $z$ & region \# 1 & region \#2 & region \# 3 & region \# 4 & region \#1 & region \#2 & region \#3 & \\
\hline Abell 1413 & 0.142 & $6.0 \pm 0.4$ & $7.8 \pm 0.7$ & $7.4 \pm 0.7$ & $5.5 \pm 0.4$ & $0.46 \pm 0.14$ & $0.41 \pm 0.16$ & $0.49 \pm 0.11$ & $0.73 \pm 0.14$ \\
\hline Abell 2204 & 0.152 & \pm 0.1 & $6.7 \pm 0.3$ & $10.2 \pm 0.7$ & $12.1 \pm 0.8$ & $0.98 \pm 0.09$ & $57 \pm 0.09$ & $0.41 \pm 0.11$ & \\
\hline Abell 665 & 0.182 & $7.7 \pm 0.7$ & $7.9 \pm 0.7$ & $8.5 \pm 0.7$ & $8.1 \pm 0.6$ & $0.32 \pm 0.12$ & $0.39 \pm 0.13$ & $0.47 \pm 0.14$ & $0.31 \pm 0.10$ \\
\hline RX J0439.0+0520 & 0.208 & $3.1 \pm 0.2$ & $4.5 \pm 0.3$ & $4.8 \pm 0.5$ & $4.2 \pm 0.5$ & $0.50 \pm 0.19$ & $0.94 \pm 0.28$ & $0.44 \pm 0.13$ & \\
\hline Abell 773 & 0.217 & $7.3 \pm 1.0$ & $9.1_{-1.3}^{+1.9}$ & $9.6_{-1.1}^{+1.8}$ & $8.1_{-1.1}^{+1.3}$ & $0.43 \pm 0.22$ & $0.27 \pm 0.23$ & $0.68 \pm 0.28$ & $0.07_{-0.07}^{+0.25}$ \\
\hline Abell 1763 & 0.223 & & $8.3 \pm 0$ & $7.0 \pm 0.3$ & $6.6 \pm 0.3$ & $0.47 \pm 0.08$ & & $0.30 \pm 0.06$ & $0.26 \pm 0.05$ \\
\hline Abell 2390 & 0.228 & $7.3 \pm 0.2$ & $9.4 \pm 0.3$ & $10.0 \pm 0.5$ & $9.7 \pm 0.4$ & $0.43 \pm 0.05$ & $0.43 \pm 0.06$ & $0.29 \pm 0.06$ & $0.17 \pm 0.05$ \\
\hline Abel & 0.253 & & $6.8 \pm 0.1$ & $7.2 \pm 0.1$ & $7.4 \pm 0.1$ & $0.46 \pm$ & $0.37 \pm 0.02$ & 0.02 & 0.23 \\
\hline Abe & 0.282 & & \pm 1.0 & \pm 1.0 & $9.1 \pm$ & .16 & & .11 & .09 \\
\hline Abe & 0.288 & & \pm 0.5 & \pm 0.6 & $6.5 \pm$ & .12 & & .11 & \\
\hline MS 100 & 0.306 & & $5.8 \pm 0.6$ & \pm 0.8 & $6.5 \pm 0.7$ & 14 & & 0.10 & \\
\hline MS 2 & .313 & & 5 & 5 & & & & .07 & \\
\hline & & & & & & & & .10 & \\
\hline ZWCL 1358+6245 & 0.328 & & .7 & & & 10 & 0.14 & 0.27 & \\
\hline RX J0256.5+0006 & .360 & & & & & & & 10 & \\
\hline RX J03 & 0.370 & 4.0 & 5.5 & 5 . & 6.3 & \pm 0.12 & & .14 & \\
\hline RX J0426.1+ & 0.380 & 4.5 & .0 & .7 & 5.0 & $0.47 \pm 0.15$ & $0.06_{-0.06}^{+0.18}$ & $0.07_{-0.07}^{+0.16}$ & 0.3 \\
\hline MACS J2228.5+2036 & 0.412 & $7.6 \pm 1.3$ & $9.9 \pm 2.2$ & $7.9 \pm 1.4$ & $6.9 \pm 0.8$ & $0.56 \pm 0.21$ & $0.26 \pm 0.19$ & $0.29 \pm 0.15$ & 0.52 \\
\hline MACS J2214.9-1359 & 0.483 & & $9.1 \pm 1.7$ & $12.7_{-2.8}^{+5.3}$ & $8.4 \pm 1.5$ & $0.27 \pm 0.21$ & $0.23 \pm 0.22$ & $0.17_{-0.17}^{+0.20}$ & \\
\hline MAC & 0.490 & & & & & & & & 0.17 \\
\hline MS 0015.9+1609 & 0.541 & & $9.9 \pm 1.0$ & $10.5_{-0.7}^{+1.5}$ & $10.3 \pm 1.1$ & $0.61 \pm 0.14$ & $0.22 \pm 0.08$ & $0.09 \pm 0.09$ & 0.09 \\
\hline MACS $1423.8+2404$ & 0.545 & & $5.8 \pm 0.2$ & $7.1 \pm 0.3$ & $6.9 \pm 0.5$ & $0.66 \pm 0.08$ & $0.48 \pm 0.07$ & $0.34 \pm 0.05$ & \\
\hline MS 0451.6-0305 & 0.530 & & $8.9 \pm 0.6$ & & & & $0.28=$ & 0.10 & \\
\hline MACS J2129.4-0741 & 0.570 & $7.0 \pm 1.1$ & $7.2 \pm 1.2$ & $7.6 \pm 1.2$ & $5.2 \pm 0.7$ & $0.45 \pm 0.24$ & $0.33 \pm 0.20$ & $0.41 \pm 0.23$ & $0.65 \pm 0.17$ \\
\hline MS 2053.7-0449 & 0.583 & $4.7 \pm 0.7$ & $4.5 \pm 0.6$ & $5.6 \pm 1.1$ & $3.4 \pm 0.6$ & $0.32 \pm 0.24$ & $0.23 \pm 0.20$ & $0.26 \pm 0.23$ & $0.59 \pm 0.41$ \\
\hline MACS J0647.7+7015 & 0.584 & $14.9_{-3.0}^{+4.5}$ & $11.7_{-2.2}^{+3.6}$ & $10.7_{-2.7}^{+1.7}$ & $9.5 \pm 1.6$ & $0.43 \pm 0.39$ & $0.40 \pm 0.25$ & $0.39 \pm 0.22$ & $0+0.18$ \\
\hline RX J1120.1+4318 & 0.600 & $5.4 \pm 0.4$ & $5.2 \pm 0.4$ & $5.0 \pm 0.4$ & $4.9 \pm 0.5$ & $0.54 \pm 0.10$ & $0.51 \pm 0.18$ & $0.26 \pm 0.12$ & $0+0.16$ \\
\hline MACS J0744.8+3927 & 0.686 & $5.3 \pm 0.6$ & $8.7 \pm 1.7$ & $9.0 \pm 1.6$ & $5.9 \pm 0.7$ & $0.97_{-0.17}^{+0.40}$ & $0.18 \pm 0.23$ & $0.20 \pm 0.18$ & $0.24 \pm 0.13$ \\
\hline MS $1137.5+6625$ & 0.782 & $7.0_{-0.7}^{+1.0}$ & $7.5 \pm 1.0$ & $8.5 \pm 1.4$ & $5.6 \pm 0.7$ & $0.43 \pm 0.18$ & $0+0.26$ & $0.38 \pm 0.21$ & $0.24 \pm 0.13$ \\
\hline RX J1716.9+6708 & 0.813 & $10.5_{-2.6}^{+3.2}$ & $4.6_{-0.5}^{+1.1}$ & $4.3 \pm 0.8$ & N/A & $0.37 \pm 0.33$ & $0.23 \pm 0.21$ & $0.88 \pm 0.27$ & $\mathrm{~N} / \mathrm{A}$ \\
\hline CL J1226.9+3332 & 0.890 & $12.7 \pm 0.8$ & $11.5 \pm 0.8$ & $10.9 \pm 0.7$ & $9.3 \pm 0.5$ & $0.34 \pm 0.11$ & $0.29 \pm 0.11$ & $0.08_{-0.08}^{+0.11}$ & $0.06_{-0.06}^{+0.13}$ \\
\hline
\end{tabular}

Setting the boundary to this temperature allows for comparably sized subsets for high and low temperature clusters, even after the two other selection effects (cooling time and redshift) are taken into account. The average radial profiles presented below are not strongly dependent on small changes $(\simeq 0.2 \mathrm{keV})$ to this boundary. 
Table 7. The eight subsets of the sample used to calculate average temperature and iron abundance profiles.

\begin{tabular}{ccccc}
\hline \hline & \multicolumn{4}{c}{ Classification of Subsets } \\
subset \# & redshift & temperature & cooling & number \\
\hline 1 & $z<0.4$ & $<6.8 \mathrm{keV}$ & Yes & $6 / 6$ \\
2 & $z<0.4$ & $\geq 6.8 \mathrm{keV}$ & Yes & $4 / 4$ \\
3 & $z<0.4$ & $<6.8 \mathrm{keV}$ & No & $3 / 3$ \\
4 & $z<0.4$ & $\geq 6.8 \mathrm{keV}$ & No & $4 / 4$ \\
5 & $z>0.4$ & $<6.8 \mathrm{keV}$ & Yes & $2 / 2$ \\
6 & $z>0.4$ & $\geq 6.8 \mathrm{keV}$ & Yes & $2 / 2$ \\
7 & $z>0.4$ & $<6.8 \mathrm{keV}$ & No & $5 / 3$ \\
8 & $z>0.4$ & $\geq 6.8 \mathrm{keV}$ & No & $9 / 7$ \\
\hline
\end{tabular}

The two values in the number column represent the number of observations and the number of unique clusters in that sample, respectively.

\subsection{Central cooling}

An important mechanism in galaxy cluster evolution is the process of radiative cooling. The cooling time for a cluster can be well approximated by Sarazin (1988) as

$t_{\text {cool }}=8.5 \times 10^{10} \mathrm{yr}\left(\frac{n_{\mathrm{p}}}{10^{-3}}\right)^{-1}\left(\frac{T_{\mathrm{g}}}{10^{8} \mathrm{~K}}\right)^{1 / 2}$

where $n_{\mathrm{p}}$ is the particle density in units of $\mathrm{cm}^{-3}$ and $T_{\mathrm{g}}$ is the temperature of the gas in Kelvin. By relating the measured temperature and luminosity to the density and performing the calculation, the cooling times for each of these clusters can be calculated. Because high densities reduce the radiative cooling time, radiative cooling is potentially significant only in the central regions of a cluster. Therefore, all cooling calculations used the temperature and luminosity measured for the innermost regions. The cooling times were calculated assuming that the density is constant within the region of zero to one-half of a core radius and that all of these clusters originally formed at a redshift of $z=2$. For the calculation of the density, the bolometric luminosity (from $0-100 \mathrm{keV}$ ) and the volume of the $0-0.5 r_{\mathrm{c}}$ region were used to determine an emissivity for the region, which can then be related to the density by Sarazin (1988) as

$e^{\mathrm{ff}}=\left(1.435 \times 10^{-27}\right) T_{\mathrm{g}}^{1 / 2} n_{\mathrm{p}}^{2} \mathrm{erg} \mathrm{cm}^{-3} \mathrm{~s}^{-1}$.

This assumes that the X-ray emission is all due to thermal bremsstrahlung and also that the density and temperature of the ICM is constant within $0.5 r_{\mathrm{c}}$. A single beta fit was used instead of a more elaborate fit, and de-projection of hydrostatic equilibrium models was not done, as was carried by Bonamente et al. (2006). The cooling time only goes as the square root of the temperature, and hence it can be seen that any contamination of the temperature determination by the outer portions of the cluster (in cooling clusters only) would be a weak effect. In comparison with the cooling times calculated in Bonamente et al. (2006), there is only one discrepancy between their results and those derived here. The cluster MACS J2129.4-0741 has a cooling time slightly smaller than its age (4.64 Gyr as compared to $4.82 \mathrm{Gyr}$ ). Since this difference is small compared to the cooling time, and it is not listed as a cooling core cluster in Bonamente et al. (2006), it is treated in this study as a non-cooling core cluster as well.

The results of these calculations are listed in Table 8. The presence of cooling core clusters leads directly to another important process: central Active Galactic Nucleus (AGN) activity. For nearby clusters with cooling cores, it has been shown that they do not cool as quickly as the theoretical cooling times suggest (Fabian 1994). AGN activity could influence the temperature as far out as $\simeq 50-100 \mathrm{kpc}$, which is, on average, approximately $0.5 r_{\mathrm{c}}$ or in the first counts profile bin. The majority of cool core clusters exhibit the radio emission or bubbles usually associated with AGN activity (Dunn \& Fabian 2006). The findings of Dunn \& Fabian (2006) also show that only a small fraction of cool core clusters show no evidence of AGN activity as either bubbles or radio sources. Therefore, it is expected that central AGN activity is generally (if not always) present in the cooling core clusters in this sample as well. For completeness, all clusters were searched for possible counterpart radio sources within one arc-minute of the cluster position. The presence of sources was done using the NVSS catalog (Condon et al. 1998), and the sources were confirmed as being associated with the cluster using NED. The clusters with likely radio counterparts are denoted with a dagger in Table 8 . The presence of central AGN activity will be assumed in discussing the results for cool core clusters.

\section{Results}

After dividing the sample into eight comparable subsets based on temperature, cooling time, and redshift, relevant average profiles were calculated and compared. These divisions had to be made so as to make valid comparisons between profiles derived from low and high redshifts. Since there is currently little evidence for evolution in galaxy clusters even at high redshifts (Matsumoto et al. 2000; Mushotzky \& Loewenstein 1997), the null hypothesis for statistical tests will be that cluster abundance and temperature profiles do not change with redshift. See also Leccardi \& Molendi $(2008 \mathrm{a}, \mathrm{b})$ for $z=0.1-0.3$ studies. Only if the statistics conclusively suggest rejecting this hypothesis will evolution be considered.

\subsection{Zero iron abundances and N/A values}

As seen in Table 6, there are several instances where the best fit iron abundance was zero. As a compromise between ignoring these points altogether and producing an un-weighted average, the derived uncertainties to these points were used as statistical weights (one over the error squared). Since these zero values were most likely due to an insufficient number of detected photons rather than a true lack of iron in the ICM, this averaging was judged to be a valid approach. As a cross check, these points were excluded from the averaging as well, and the results were all within $1 \sigma$ of each other in both weighted and unweighted averages (see Sect. 4.2). Also, there was one instance where the measurement failed to converge for both the temperature and iron abundance simultaneously, and no measurement errors could be calculated. This measurement is listed with a "N/A" in Table 6.

\subsection{Averaging procedures}

In order to make the most robust study of the data, two averaging procedures (weighted and unweighted) were done on each subset listed in the following sections. The primary averaging process used was a weighted average that took into account the measurement error on each observation. For a given sample of $N$ clusters, the weighted temperature/iron abundance average $\bar{M}$ 
Table 8. General information for each cluster: the overall (defined here to be from $0-2 r_{\mathrm{c}}$ ) temperature and iron abundance, the overall luminosity, the total number of counts in the cluster, the calculations relevant to the cooling time, and finally the subset as defined in Table 7.

\begin{tabular}{|c|c|c|c|c|c|c|c|c|c|c|}
\hline \multicolumn{6}{|c|}{ Temperatures and Iron abundances for each full cluster } & \multicolumn{4}{|c|}{ Cooling calculations for each central region } & \multirow{2}{*}{$\begin{array}{c}\text { Analysis Subset } \\
\text { subset \# }\end{array}$} \\
\hline cluster name & $z$ & temperature & iron abundance & \# of Counts & $\mathcal{L}_{0-2}$ & $\mathcal{L}_{0-0.5}$ & proton density & cooling time & age & \\
\hline Abell $1413^{\dagger}$ & 0.142 & $7.0 \pm 0.3$ & $0.46 \pm 0.08$ & 11164 & 5.11 & 1.95 & 0.019 & 3.79 & 8.55 & 2 \\
\hline Abell $2204^{\dagger}$ & 0.152 & $4.3 \pm 0.1$ & $0.86 \pm 0.08$ & 16382 & 5.17 & 1.09 & 0.22 & 0.24 & 8.44 & 1 \\
\hline Abell $665^{\dagger}$ & 0.182 & $8.0 \pm 0.4$ & $0.40 \pm 0.08$ & 12526 & 3.89 & 0.87 & 0.012 & 6.91 & 8.11 & 2 \\
\hline RX J0439.0+0520 & 0.208 & $3.6 \pm 0.2$ & $0.76 \pm 0.17$ & 2018 & 1.96 & 0.75 & 0.08 & 0.57 & 7.83 & 1 \\
\hline Abell 773 & 0.217 & $8.0 \pm 0.7$ & $0.32 \pm 0.13$ & 8351 & 9.24 & 5.24 & 0.0058 & 14.72 & 7.74 & 4 \\
\hline Abell 1763 & 0.223 & $7.9 \pm 0.2$ & $0.34 \pm 0.04$ & 48845 & 8.81 & 3.52 & 0.0062 & 12.93 & 7.68 & 4 \\
\hline Abell $2390^{\dagger}$ & 0.228 & $8.5 \pm 0.2$ & $0.43 \pm 0.04$ & 36705 & 10.70 & 3.41 & 0.038 & 1.94 & 7.63 & 2 \\
\hline Abell $1835^{\dagger}$ & 0.253 & $6.0 \pm 0.1$ & $0.39 \pm 0.01$ & 242214 & 18.20 & 7.20 & 0.088 & 0.71 & 7.38 & 1 \\
\hline Abell 697 & 0.282 & $9.7 \pm 0.7$ & $0.35 \pm 0.08$ & 13442 & 16.6 & 7.75 & 0.0082 & 11.15 & 7.10 & 4 \\
\hline Abell $611^{\dagger}$ & 0.288 & $7.0 \pm 0.3$ & $0.31 \pm 0.06$ & 15213 & 6.53 & 2.64 & 0.019 & 3.92 & 7.04 & 2 \\
\hline MS 1008.1-1224 & 0.306 & $6.3 \pm 0.4$ & $0.35 \pm 0.08$ & 7257 & 4.18 & 1.28 & 0.0082 & 8.49 & 6.88 & 3 \\
\hline MS 2137.3-2353 & 0.313 & $4.6 \pm 0.1$ & $0.35 \pm 0.04$ & 16958 & 8.29 & 2.87 & 0.10 & 0.60 & 6.81 & 1 \\
\hline Abell 1995 & 0.319 & $8.9 \pm 0.5$ & $0.28 \pm 0.10$ & 34062 & 15.20 & 5.73 & 0.0062 & 13.58 & 6.73 & 4 \\
\hline ZWCL $1358+6245^{\dagger}$ & 0.328 & $6.1 \pm 0.3$ & $0.39 \pm 0.08$ & 8208 & 3.37 & 0.94 & 0.035 & 1.65 & 6.68 & 1 \\
\hline RX J0256.5+0006 & 0.360 & $5.0 \pm 0.2$ & $0.34 \pm 0.06$ & 8608 & 4.49 & 1.36 & 0.0061 & 11.32 & 6.40 & 3 \\
\hline RX J0318.2-0301 & 0.370 & $5.7 \pm 0.3$ & $0.28 \pm 0.08$ & 7879 & 3.76 & 1.31 & 0.0064 & 9.16 & 6.32 & 3 \\
\hline RX J0426.1+1655 & 0.380 & $5.4 \pm 0.4$ & $0.19 \pm 0.07$ & 33174 & 1.55 & 0.77 & 0.021 & 2.83 & 6.23 & 1 \\
\hline MACS J2228.5+2036 & 0.412 & $7.1 \pm 0.5$ & $0.33 \pm 0.10$ & 6199 & 15.29 & 6.24 & 0.0081 & 10.30 & 5.97 & 8 \\
\hline MACS J2214.9-1359 & 0.483 & $8.8 \pm 0.8$ & $0.29 \pm 0.12$ & 3399 & 14.36 & 5.20 & 0.011 & 7.61 & 5.43 & 8 \\
\hline MACS J1311.0-0310† & 0.490 & $6.5 \pm 0.6$ & $0.45 \pm 0.13$ & 2111 & 10.57 & 4.60 & 0.021 & 3.09 & 5.38 & 5 \\
\hline MS 0015.9+1609 & 0.541 & $9.8 \pm 0.6$ & $0.28 \pm 0.06$ & 17837 & 23.57 & 3.28 & 0.0041 & 21.99 & 5.02 & 8 \\
\hline MACS $1423.8+2404^{\dagger}$ & 0.545 & $5.3 \pm 0.1$ & $0.54 \pm 0.05$ & 17674 & 10.06 & 3.31 & 0.12 & 0.50 & 4.99 & 5 \\
\hline MS 0451.6-0305 & 0.550 & $9.4 \pm 0.7$ & $0.40 \pm 0.11$ & 14303 & 22.11 & 7.34 & 0.011 & 8.32 & 4.96 & 8 \\
\hline MACS J2129.4-0741 & 0.570 & $7.0 \pm 1.1$ & $0.41 \pm 0.23$ & 1755 & 10.63 & 2.59 & 0.018 & 4.64 & 4.82 & 6 \\
\hline MS 2053.7-0449 & 0.583 & $4.9 \pm 0.5$ & $0.28 \pm 0.10$ & 1733 & 1.95 & 0.41 & 0.0083 & 7.75 & 4.74 & 7 \\
\hline MACS J0647.7+7015 & 0.584 & $11.5_{-2.0}^{+2.8}$ & $0.19_{-0.19}^{+0.26}$ & 2717 & 16.27 & 7.58 & 0.013 & 8.79 & 4.73 & 8 \\
\hline RX J1120.1+4318 & 0.600 & $5.2 \pm 0.4$ & $0.37 \pm 0.11$ & 10239 & 7.22 & 3.28 & 0.0063 & 11.21 & 4.63 & 7 \\
\hline MACS J0744.8+3927† & 0.686 & $6.8 \pm 0.7$ & $0.51 \pm 0.18$ & 1153 & 9.36 & 2.66 & 0.063 & 1.09 & 4.11 & 6 \\
\hline MS 1137.5+6625 & 0.782 & $7.2 \pm 0.6$ & $0.26 \pm 0.13$ & 4073 & 6.03 & 2.07 & 0.014 & 5.61 & 3.59 & 8 \\
\hline RX J1716.9+6708 & 0.813 & $4.6 \pm 0.5$ & $0.57 \pm 0.23$ & 1649 & 6.16 & 2.21 & 0.0088 & 9.69 & 3.43 & 7 \\
\hline CL J1226.9+3332 & 0.890 & $11.1 \pm 0.3$ & $0.20 \pm 0.05$ & 29846 & 20.56 & 10.06 & 0.014 & 7.42 & 3.07 & 8 \\
\hline
\end{tabular}

The column $\mathcal{L}_{0-0.5}$ is the luminosity from $0-0.5 r_{\mathrm{c}}$, and $\mathcal{L}_{0-2}$ is the luminosity from $0-2 r_{\mathrm{c}}$. The luminosities are in erg $-\mathrm{s}^{-1}$, the times are in Gyr, and the densities are in units of $\mathrm{cm}^{-3}$. Galaxy clusters with a dagger $(\dagger)$ next to them have confirmed radio sources likely associated with the cluster. The temperature and iron abundance have the same units as in Table 6.

is calculated using the measurements $m_{i}$ and the measurement error $\sigma_{i}$ as

$\bar{M}=\frac{\sum_{i=1}^{N} \frac{m_{i}}{\sigma_{i}^{2}}}{\sum_{i=1}^{N} \frac{1}{\sigma_{i}^{2}}} \pm\left(\sum_{i=1}^{N} \frac{1}{\sigma_{i}^{2}}\right)^{-1 / 2}$

As a check to ensure that no significant biases arise due to the weighting procedure, a standard (unweighted) mean and standard deviation on that mean were also calculated, both of which only depend on the measurements themselves and not the error on those measurements. The unweighted averages further demonstrate the robustness of these measurements, as the choice of weighted or unweighted averaging does not affect the basic conclusions.

\subsection{Average profiles}

The average temperature and iron abundance profiles are written out in Tables 9 and 10. They are organized by subset and radial region, and show the unweighted values in parentheses next to the weighted averages. Since the weighted mean emphasizes measurements with the smallest errors and there was substantial variance in the quality of the spectra, the weighted mean was chosen to be the basis for the subsequent figures and discussion. However, both means exhibited the same general trends and results.

\subsection{Temperature profiles}

The temperature profiles of Table 9 suggest that the overall temperature and cooling time completely define the temperature profiles of clusters. The average temperature profiles for cooling core clusters exhibit a clear increase in temperature with radius, while clusters without cool cores are either isothermal or have temperatures that decrease with radius. Similar temperature profiles for non-cool core clusters has been confirmed by Sanderson et al. (2006) and Leccardi \& Molendi (2008b).

There are also no differences between the temperature profiles of high and low temperature clusters of comparable cooling times with the exception of an overall offset due to the higher overall temperatures. Because the temperature profiles do not seem to deviate from expectations based on their overall temperature and cooling times, the discussion in Sect. 5 will focus primarily on the measured iron abundance profiles.

\subsection{Iron profiles}

The iron abundance radial profiles are now taken into consideration. 
Table 9. Average temperature as a function of counts radius, separated by redshift, temperature and cooling times. Unweighted averages are in parentheses.

\begin{tabular}{ccccc}
\hline \hline & & \multicolumn{2}{c}{ Temperatures in keV } & \\
subset \# & region \#1 & region \#2 & region \#3 & region \#4 \\
\hline 1 & $4.67(4.18) \pm 0.04(0.27)$ & $6.51(6.38) \pm 0.07(0.63)$ & $7.05(6.92) \pm 0.08(0.87)$ & $7.26(7.32) \pm 0.09(1.23)$ \\
2 & $7.06(6.91) \pm 0.16(0.37)$ & $8.37(8.04) \pm 0.24(0.50)$ & $8.68(8.36) \pm 0.29(0.60)$ & $7.51(7.45) \pm 0.24(0.92)$ \\
3 & $4.81(5.15) \pm 0.23(0.57)$ & $5.54(5.55) \pm 0.31(0.11)$ & $5.0(5.69) \pm 0.29(0.89)$ & $4.18(5.52) \pm 0.22(0.91)$ \\
4 & $7.81(8.13) \pm 0.28(0.49)$ & $8.69(9.12) \pm 0.35(0.28)$ & $7.34(8.61) \pm 0.24(0.59)$ & $6.90(8.15) \pm 0.24(0.57)$ \\
5 & $4.11(4.38) \pm 0.11(0.30)$ & $5.81(6.26) \pm 0.18(0.49)$ & $7.16(7.29) \pm 0.28(0.15)$ & $6.08(5.89) \pm 0.38(1.06)$ \\
6 & $5.65(6.13) \pm 0.52(0.85)$ & $7.68(7.95) \pm 0.96(0.79)$ & $8.12(8.27) \pm 0.97(0.68)$ & $5.56(5.55) \pm 0.51(0.31)$ \\
7 & $5.34(6.86) \pm 0.32(1.81)$ & $4.93(4.78) \pm 0.33(0.23)$ & $4.94(4.97) \pm 0.36(0.38)$ & $4.21(2.75) \pm 0.37(1.44)$ \\
8 & $9.47(9.89) \pm 0.37(1.09)$ & $9.48(9.78) \pm 0.37(0.55)$ & $9.83(10.03) \pm 0.40(0.63)$ & $7.90(8.18) \pm 0.29(0.63)$ \\
\hline
\end{tabular}

Table 10. Average iron abundance as a function of counts radius, separated by redshift, temperature and cooling times. Unweighted averages are in parentheses.

\begin{tabular}{ccccc}
\hline \hline & & \multicolumn{2}{c}{ Iron Abundance in Solar Units } & region \#4 \\
subset \# & region \#1 & region \#2 & region \#3 & $0.25(0.32) \pm 0.02(0.09)$ \\
\hline 1 & $0.48(0.55) \pm 0.02(0.09)$ & $0.37(0.45) \pm 0.02(0.12)$ & $0.27(0.30) \pm 0.02(0.05)$ & $0.27(0.39) \pm 0.04(0.12)$ \\
2 & $0.42(0.42) \pm 0.04(0.03)$ & $0.38(0.37) \pm 0.05(0.04)$ & $0.33(0.37) \pm 0.05(0.07)$ & $0.27(0.0 .24) \pm 0.06(0.10)$ \\
3 & $0.46(0.45) \pm 0.08(0.07)$ & $0.34(0.34) \pm 0.07(0.04)$ & $0.28(0.27) \pm 0.06(0.05)$ & $0.13(0.24) \pm 0.05(0.06)$ \\
4 & $0.44(0.43) \pm 0.06(0.04)$ & $0.20(0.25) \pm 0.05(0.07)$ & $0.37(0.47) \pm 0.05(0.09)$ & $0.27(0.25) \pm 0.04(0.07(0.13)$ \\
5 & $0.56(0.43) \pm 0.07(0.24)$ & $0.51(0.92) \pm 0.07(0.44)$ & $0.32(0.22) \pm 0.05(0.13)$ & $0.43(0.35) \pm 0.07(0.13)$ \\
6 & $0.66(0.71) \pm 0.19(0.26)$ & $0.26(0.26) \pm 0.15(0.08)$ & $0.28(0.31) \pm 0.14(0.11)$ & $0.39(0.45) \pm 0.10(0.21)$ \\
7 & $0.50(0.41) \pm 0.09(0.07)$ & $0.34(0.32) \pm 0.11(0.09)$ & $0.34(0.47) \pm 0.10(0.21)$ & $0.08(0.20) \pm 0.15(0.20)$ \\
8 & $0.47(0.47) \pm 0.06(0.05)$ & $0.25(0.24) \pm 0.05(0.05)$ & $0.16(0.22) \pm 0.05(0.05)$ & $0.22(0.23) \pm 0.05(0.08)$ \\
\hline
\end{tabular}

\subsubsection{Low temperature, cool core clusters}

The first subsets to consider are those of low temperature, cool core clusters. These clusters have been shown to have strong iron abundance gradients (De Grandi et al. 2004) at low redshifts and are often associated with central AGN activity. Not only do the two subsets considered here (subsets \# 1 and 5) show no evidence for evolution with redshift, the iron abundance profile is almost identical to the profiles of still lower $(z \leq 0.1)$ redshift clusters discussed in De Grandi et al. (2004). The results of De Grandi et al. (2004) have been re-projected to the length scale used here and corrected for the differences in their solar abundance model. All three profiles are shown in Fig. 1. From Fig. 1 there is no evidence for evolution in the iron abundance of cool core clusters from $\langle z\rangle \simeq 0.7$ to $\langle z\rangle \simeq 0$. It should also be noted in Table 10 that low temperature cool core clusters (subsets 1 and 5) do not have statistically significant differences in overall iron abundance or in average iron abundance profiles versus redshift. The same lack of difference is also seen when comparing high temperature cool core clusters (subsets 2 and 6).

\subsubsection{High temperature, cool core clusters}

There are only two clusters in the high redshift subset for clusters with high temperatures and cool cores (subset \#6) which is too small of a sample to be considered representative of the entire population. However, it should nevertheless be noted that the average iron abundance profile for this small subset is entirely consistent point-for-point with the results of the larger low redshift sample. As can be seen in Fig. 2, the average profile in both cases has a higher central abundance, with evidence for a decreasing gradient outward. Even though the average profile for the high redshift sample may not represent the population, this small sample still shows no evidence for evolution in the iron abundance profile with redshift.

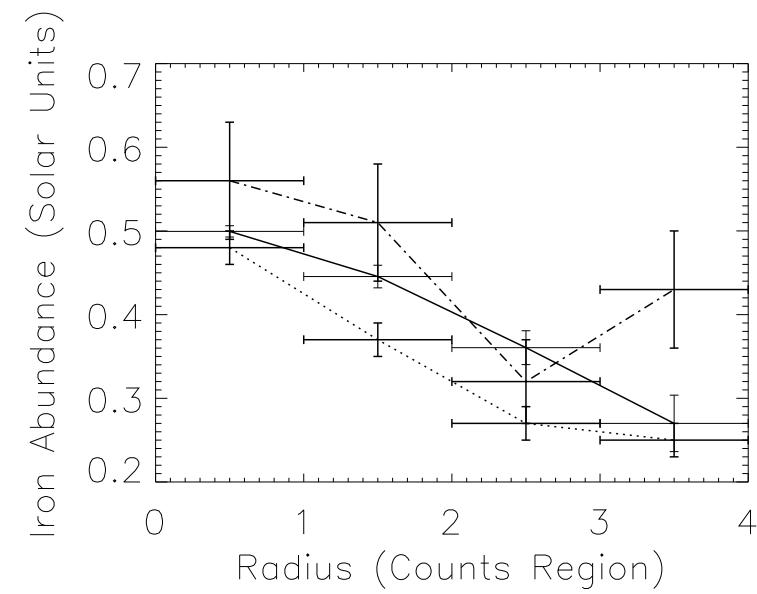

Fig. 1. Iron abundance as a function of radius for clusters with low temperatures and short cooling times, separated by redshift. The dotted line corresponds to subset \#1 $(z<0.4)$ while the dashed line corresponds to subset \#5 ( $z>0.4)$. The solid line is from De Grandi et al. (2004).

\subsubsection{Low temperature, non-cool core clusters}

Subsets \#3 and 7 describe the average profiles for low temperature clusters without cool cores. Although both of these samples are small, Fig. 3 shows that the two profiles are very consistent with one another.

\subsubsection{High temperature, non-cool core clusters}

Both subsets in this case have a relatively high number of clusters. Again in these two subsets, the two radial profiles are wholly consistent with one another, with no reason to believe that the iron abundance in these subsets is changing with redshift. All of the analysis suggests that the iron abundance is not 


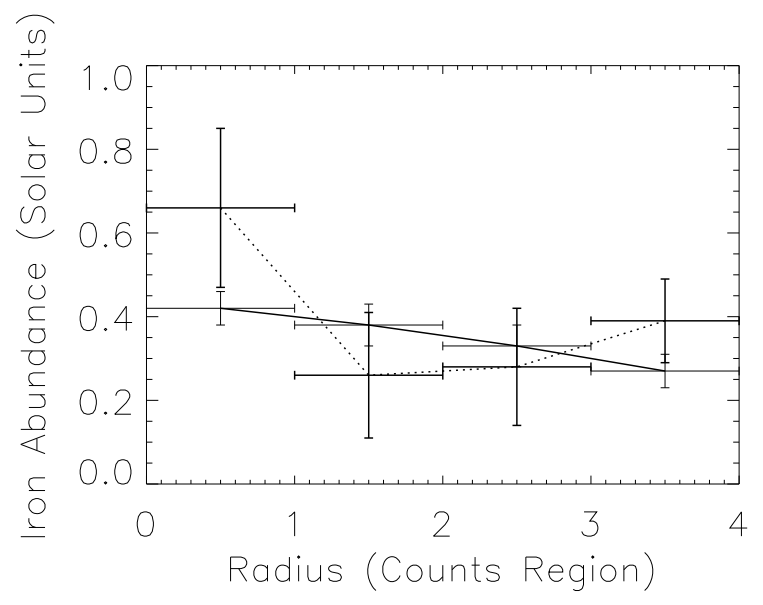

Fig. 2. Iron abundance as a function of radius for clusters with high temperatures and short cooling times, separated by redshift. The solid line corresponds to subset \#2 $(z<0.4)$. The dashed line corresponds to subset \#6 $(z>0.4)$.

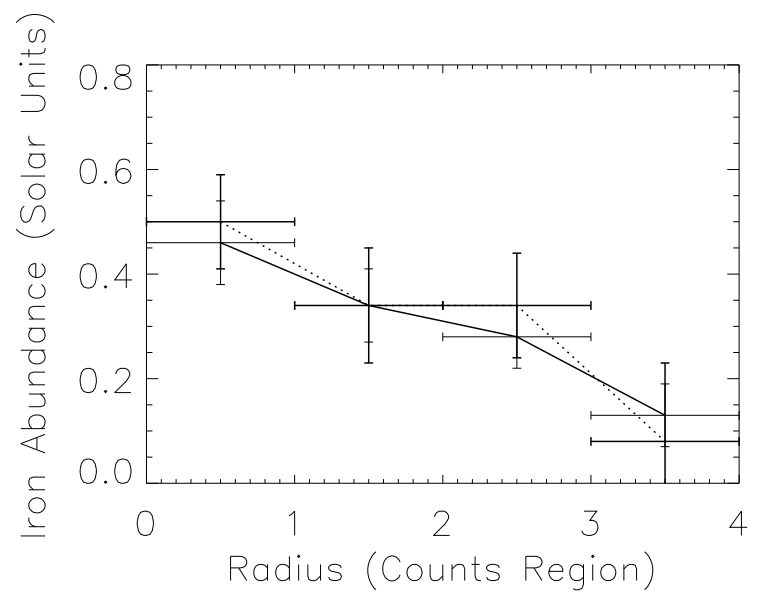

Fig. 3. Iron abundance as a function of radius for clusters with low temperatures and long cooling times, separated by redshift. The solid line corresponds to subset \#3 $(z<0.4)$. The dashed line corresponds to subset \#7 $(z>0.4)$.

evolving with redshift independent of the temperature and cooling time.

\section{Discussion}

\subsection{The data analysis}

Before discussing the radial dependencies of the iron abundance and the temperature, it is worthwhile to review why and how the data from the clusters were averaged together in the manner that was done. As noted in Sects. 2.3 and 2.4, two different radii/scales were chosen. The first was the core radius derived from a single $\beta$ model. This radius corresponds directly to the dynamical scale of the cluster. This is the natural choice for a scale length here, as it is based on the dynamics of the cluster.

A second scale length was used to find four radial regions with approximately equal numbers of net counts. Although this scale is independent of the dynamics, it is useful for testing whether the profiles are sensitive to the scale length chosen. This "equal counts per enclosed area" process was chosen for its simplicity in calculation, and for its ability to increase the significance of measurements in the outer regions of the clusters. When

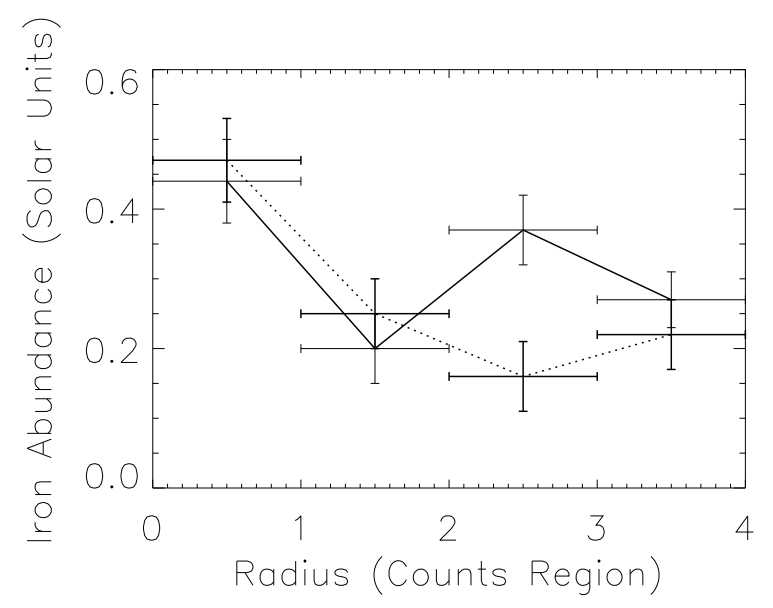

Fig. 4. Iron abundance as a function of radius for high temperature clusters with long cooling times, separated by redshift. The solid line corresponds to subset \#4 $(z<0.4)$. The dashed line corresponds to subset \#8 $(z>0.4)$.

the counts and core radii were converted into a linear projected length scale such as Mpc, they cover very similar ranges. The main inconsistency occurs in the outermost regions, where the counts radius extends much further outward than the core radius. Therefore, overall consistency between the results derived by the two different methods was expected. Since the counts radius is so strongly correlated with the core radius and offers better statistics across each cluster, the results of the core radius measurements have been omitted from the text even though they are wholly consistent with these results.

Another possible choice of length scale is the virial radius of the cluster, but the easiest way of estimating $r_{\text {virial }}$ (or $r_{200}$ ) relates directly to the overall temperature (e.g., Jones et al. 2003). If the virial radius was used in this case, the temperature would be correlated with the radial regions. Because of the $L_{\mathrm{x}, \mathrm{bol}}-T$ relationship (e.g. Ota et al. 2006) and clusters at higher redshifts must be on average more luminous to be detected with a sufficient statistical significance, the scale radius chosen needs to take into account this selection effect. Because the virial radius is related to the overall temperature, and therefore luminosity, it is difficult to correct for this selection effect. As an example, there exist nearby clusters such as Perseus (Arnaud et al. 1994) and Coma (e.g., Watanabe et al. 1999; De Grandi et al. 2004; Adami et al. 2006) with comparable X-ray luminosities that exhibit very different temperature and iron abundance profiles.

As a further check of our analysis, our results were compared with other published measurements such as Kotov \& Vikhlinin (2005) for CL 0016+16 (MS 0015.9+1609), and the temperature/abundance results published here tend to be higher by $\sim 1-1.5 \sigma$ than those of Kotov \& Vikhlinin (2005). The work of Balestra et al. (2007) shows how using an updated analysis tool can bring out differences of this scale. Furthermore, the differences may be due to systematics between the Chandra and XMM-Newton analysis chains as well as the statistics between the two observations themselves. These differences in the core radius measurements are on the order of about $20 \%$ which should not strongly affect the results of the average profiles. The measured profiles of Kotov \& Vikhlinin (2005) also measure the radial profile in a different energy band than our measurement (0.5-2 keV in Kotov \& Vikhlinin (2005) versus $0.5-10 \mathrm{keV}$ in this work). Three other clusters were studied by Snowden et al. (2008), and these results are consistent within 10-20\% and 
usually within 1 standard deviation. An important caveat is that Snowden et al. (2008) used the MOS cameras of XMM-Newton, whereas Chandra was used in this study for measuring the temperature of Abell 1413 and Abell 2204. The systematic differences in response and sensitivity may be responsible for the discrepancies between the results of Snowden et al. (2008) and those listed here.

The results of the counts profiles results will now be taken at face value, and the physical interpretation of noticeable variations will be discussed.

\subsection{Review and comparison with previous work on the chemical evolution of the ICM}

Balestra et al. (2007), Maughan et al. (2008) and Werner et al. (2008) all discuss evidence for the chemical evolution of the ICM. Older works (e.g., Mushotzky \& Loewenstein 1997; Rizza et al. 1998) did not report any statistically significant evidence for chemical evolution with redshift. The recent work that found the strongest evidence for evolution is Balestra et al., but this evolution was found only when data based on all the $z<1$ clusters in their sample were combined (compare with the smaller previous set in their Fig. 3). Furthermore, the derived chemical evolution required the use of the formal statistical best fit uncertainty instead of the observed dispersions (e.g. their Fig. 14). In contrast, for a similar redshift range (below about 0.9 ), the results of Maughan et al. are consistent with no evolution.

From the theoretical point of view as described in Kapferer et al. (2007), Sarazin (1988), and references therein, the chemical evolution of the ICM is a complex combination of effects due to cluster merging, infall of enriched material, galactic ram pressure stripping, galactic winds, and other possible causes. For the relaxed clusters considered in this paper Kapferer et al. (2007) predicted no observable chemical evolution from redshift 0.15 to 0.9 .

Our paper shows how it is important to compare like clusters and that there is room for further theoretical studies to understand the apparent lack of evolution of the radial dependence of the enriched material of the ICM, and at the same time heating via infall of metal rich gas clouds.

\subsection{Scenarios for heating and iron enrichment}

The data presented here are consistent with no evolution in radial profiles of iron abundance and temperature. The modeling of this should be consistent with the concept of hierarchical formation of structure in the universe (e.g. Gao et al. 2008, and references therein). Because the iron abundance does not appear to be strongly correlated to the overall temperature, gravitational infall is the most likely scenario for heating clusters beyond $\simeq 6.5 \mathrm{keV}$. This infall must take place in such a manner as not to change the radial profile of iron abundance or temperatures. For noncooling clusters this means the clusters somehow have no measurable temperature gradients inside about $500 \mathrm{kpc}$ but maintain their abundance gradients while (presumably) increasing in temperature in a hierarchical growth model. In order to demonstrate why infall is the preferred major energy input, the process of heating has to be considered in some detail.

An estimate of the amount of near solar abundance material that is added along with the energy to boost the $\langle k T\rangle$ from $4 \mathrm{keV}$ to $8 \mathrm{keV}$ is made now. We assume typical $L_{\mathrm{X}}-T$ (Ota et al. 2006; Ettori et al. 2004b; Stanek et al. 2006) and $L_{\mathrm{X}}-M$ (e.g. Rykoff et al. 2008; Stanek et al. 2006) relations apply to the clusters studied here. Under these assumptions, if the temperature increases by a factor of 2 then the luminosity increases by at least a factor of 4 and the mass by a factor of 3 , the amount of mass added to the clusters being on the order of $3 \times 10^{14} M_{\odot}$. Since galaxies are thought to make up only about $1 / 5$ of the baryonic mass in clusters (see Loewenstein 2006, and references there in), it is implausible that this mass and additional gravitational energy is added by normal galaxies. This mass and gravitational energy must come instead from the infall of atypical galaxies that have gas masses that greatly exceed their stellar masses or gas clouds that never formed into galaxies. Damped Lyman $\alpha$ absorbers (DLAs) have metallicities that are near solar at $z$ of 1 or higher (Meiring et al. 2007, and references therein) which makes the ability to add both mass and metals via infalling clouds plausible. It is beyond the scope of this work, though, to carry out detailed calculations of this infall scenario. However, an implication of the estimates made here is that sight lines on the outskirts of clusters should show the presence of DLAs at the cluster redshift. Furthermore, if mass is added with energy as implied by the $L-T$ and $L-M$ relationships used here, this rules out processes which might provide energy and metals but not significant amounts of additional mass, such as SN or AGNs.

Instead, suppose that the $L-M$ relationship used here doesn't apply to these high $z$ clusters. In this case, supernovae would still not be a plausible explanation. This is because the energy input of approximately $10^{64} \mathrm{erg}$ would require an unreasonably high number of $\mathrm{SNe}$ when all the available energy is transferred to the ICM (Conroy \& Ostriker 2008). For example, suppose there are 1000 galaxies per cluster. This translates into $10^{10} \mathrm{SNe}$ per galaxy or $10^{1} \mathrm{SNe} /$ year for $10^{9}$ years. It is also implausible that a central AGN could provide this much heat $\left(10^{64} \mathrm{erg}\right)$, as this heat would require $10^{55} \mathrm{erg} /$ year deposited for $1 \mathrm{~Gy}$, or $10^{47} \mathrm{erg} \mathrm{s}^{-1}$ minimum energy generation assuming $100 \%$ efficiency in transfering energy to heat. Since the magnitude of heating seems beyond what central AGN activity could provide, an infall scenario is a more likely explanation.

\subsection{Scenarios for mixing}

Since there is no evidence for evolution in every type of cluster considered in this study (as seen in the figures), it will be assumed for the sake of discussion that clusters are not mixing over this range of redshifts.

Beginning with the cool core clusters in Fig. 1, it is seen that they exhibit almost no signs of evolution in their iron abundance profiles from the $0.4-0.9$ redshift bin up to the present day, even though there is a very clear gradient at all redshifts. This suggests two possible scenarios: either enrichment and mixing both exist in such a way that neither is dominant, or neither process occurs. If neither process occurs, the unchanging iron abundance profile can be explained as being due to the average cluster galaxy having lost most of its gas by $z \sim 0.8$. In this case the galaxies have no gas to stir up and mix the ICM. An absence of gas in cluster galaxies would also manifest itself in a low, non-evolving star formation rate (Homeier et al. 2005). A low star formation rate occurs if ram pressure stripping has removed the majority of the gas from most of the cluster galaxies before redshift $z=0.8$ within $500 \mathrm{kpc}$ of the cluster center, well within the regions observed in this study. The suggestion that most gas is stripped from galaxies by $z \simeq 0.4$ was also seen by Butcher \& Oemler (1984); Dressler et al. (1997).

As the gradient in iron abundance has remained constant with redshift, there is also no evidence for growth in the gradient. Substantial changes in the abundance gradient would be 
expected if there was continuous activity from the central AGN. Therefore, energy input to the ICM beyond gravitational infall (Bode et al. 2007) must not have caused appreciable iron enrichment.

For non-cool core and high temperature clusters, there is less statistical evidence to suggest that the iron abundance decreases with radius in the same manner as low temperature cool-core clusters. However, as noted above, it is assumed there is no evolution in the radial profiles of the ICM with redshift. In this case, galactic motion within the cluster does not result in ram pressure stripping, one of the key processes for iron enrichment (and possibly mixing) in the ICM. Without ram pressure stripping from galaxies to enrich or mix the ICM, the iron abundance (and iron abundance profile) of the ICM remains static over long times.

\section{Summary and conclusions}

Average radial profiles of the temperature and iron abundance of $\mathrm{X}$-ray bright clusters in the redshift range from about 0.14 to 0.9 were calculated. We find no evidence for evolution within similar sets of clusters. The total element abundance remains constant with decreasing $z$ and the gradients remain approximately the same. These observations suggest that gravitational infall is the dominant mechanism for heating of the ICM, and also that ram pressure stripping does not substantially change the ICM out to large redshifts. If clusters experience a significant (factor of 2 or more) amount of gravitational mass infall, then the iron abundance profile remains constant while the overall temperature increases. This infall needs to simultaneously keep the profile (within error of about $20 \%$ ) isothermal while also maintaining the same iron abundance profile. Mass and energy considerations suggest infall of metal rich gas, but how the process takes place needs further theoretical study. These same considerations of mass and energy suggest that although central AGN activity is directly related to cooling cores, the central AGN does not produce significant amounts of metal rich gas and energy to the ICM beyond its local environment of about 50-100 kpc.

Other theoretical challenges to consider include how clusters formed in the first place with both abundance gradients and isothermal temperature profiles. Work also needs to be done on how the frequency and scale of merger activity (e.g. Wik et al. 2008) changes the abundance and temperature gradients of clusters. Another potentially important aspect of modeling galaxy clusters theoretically appears to be an accurate modeling of the local environment surrounding the cluster, as the infall of material initially outside of the cluster could play an important role in its evolution.

From an observational point of view it will be interesting to measure temperature and abundance gradients for clusters at $z$ greater than 0.9 as well as make measurements of DLAs by means of QSOs whose sight lines are at $\lesssim 1 \mathrm{Mpc}$ distances from the centers of rich clusters. These measurements may help determine more conclusively whether galaxy clusters are evolving in the ways described here and what kind of material might be falling in to cause the highest temperature clusters of galaxies.

Acknowledgements. This research has made use of the NASA/IPAC Extragalactic Database (NED) which is operated by the Jet Propulsion Laboratory, California Institute of Technology, under contract with the National Aeronautics and Space Administration. We would like to thank the Max Planck
Institut für Kernphysik in Heidelberg, Germany and Le Laboratoire d'Astrophysique de Marseille in Marseille, France for their hospitality. We would also like to thank A. Kathy Romer and Kivanc Sabirli for providing processed XMM data used in the early stages of this analysis. We would also like to thank Wilifried Domainko for his review and suggestions for improvement. We thank the referee for many insightful comments that greatly improved the manuscript. Finally, we would like to acknowledge support from NASA Illinois Space Grant NGT5-40073.

\section{References}

Adami, C., Picat, J. P., Savine, C., et al. 2006, A\&A, 451, 1159

Allen, S. W., Schmidt, R. W., \& Fabian, A. C. 2002, MNRAS, 335, 256 Anders, E., \& Grevesse, N. 1989, Geochim. Cosmochim. Acta, 53, 197

Arnaud, M. 2005, in Background Microwave Radiation and Intracluster Cosmology, ed. F. Melchiorri, \& Y. Rephaeli, 77

Arnaud, K. A., Mushotzky, R. F., Ezawa, H., et al. 1994, ApJ, 436, L67

Balestra, I., Tozzi, P., Ettori, S., et al. 2007, A\&A, 462, 429

Baumgartner, W. H., Loewenstein, M., Horner, D. J., \& Mushotzky, R. F. 2005, ApJ, 620, 680

Bode, P., Ostriker, J. P., Weller, J., \& Shaw, L. 2007, ApJ, 663, 139

Bonamente, M., Joy, M. K., LaRoque, S. J., et al. 2006, ApJ, 647, 25

Butcher, H., \& Oemler, Jr., A. 1984, ApJ, 285, 426

Condon, J. J., Cotton, W. D., Greisen, E. W., et al. 1998, AJ, 115, 1693

Conroy, C., \& Ostriker, J. P. 2008, ApJ, 681, 151

De Grandi, S., Ettori, S., Longhetti, M., \& Molendi, S. 2004, A\&A, 419, 7

Domainko, W., Mair, M., Kapferer, W., et al. 2006, A\&A, 452, 795

Dressler, A., Oemler, A. J., Couch, W. J., et al. 1997, ApJ, 490, 577

Dunn, R. J. H., \& Fabian, A. C. 2006, MNRAS, 373, 959

Ettori, S., De Grandi, S., \& Molendi, S. 2002, A\&A, 391, 841

Ettori, S., Tozzi, P., Borgani, S., \& Rosati, P. 2004a, A\&A, 417, 13

Ettori, S., Tozzi, P., Borgani, S., \& Rosati, P. 2004b, A\&A, 417, 13

Fabian, A. C. 1994, ARA\&A, 32, 277

Fabian, A. C., Sanders, J. S., Taylor, G. B., et al. 2006, MNRAS, 366, 417

Gao, L., Navarro, J. F., Cole, S., et al. 2008, MNRAS, 387, 536

Gitti, M., \& Schindler, S. 2005, Adv. Space Res., 36, 613

Homeier, N. L., Demarco, R., Rosati, P., et al. 2005, ApJ, 621, 651

Jones, L. R., Ponman, T. J., Horton, A., et al. 2003, MNRAS, 343, 627

Kapferer, W., Kronberger, T., Weratschnig, J., et al. 2007, A\&A, 466, 813

Kotov, O., \& Vikhlinin, A. 2005, ApJ, 633, 781

Leccardi, A., \& Molendi, S. 2008a, A\&A, 487, 461

Leccardi, A., \& Molendi, S. 2008b, A\&A, 486, 359

Loewenstein, M. 2006, ApJ, 648, 230

Matsumoto, H., Tsuru, T. G., Fukazawa, Y., Hattori, M., \& Davis, D. S. 2000, PASJ, 52, 153

Maughan, B. J., Jones, C., Forman, W., \& Van Speybroeck, L. 2008, ApJS, 174, 117

Meiring, J. D., Lauroesch, J. T., Kulkarni, V. P., et al. 2007, MNRAS, 376, 557

Mushotzky, R. F., \& Loewenstein, M. 1997, ApJ, 481, L63

Nousek, J. A., \& Shue, D. R. 1989, ApJ, 342, 1207

Ota, N., Kitayama, T., Masai, K., \& Mitsuda, K. 2006, ApJ, 640, 673

Ota, N., Murase, K., Kitayama, T., et al. 2008, A\&A, 491, 363

Pacaud, F., Pierre, M., Adami, C., et al. 2007, MNRAS, 382, 1289

Rizza, E., Burns, J. O., Ledlow, M. J., et al. 1998, MNRAS, 301, 328

Rosati, P., Borgani, S., \& Norman, C. 2002, ARA\&A, 40, 539

Rykoff, E. S., Evrard, A. E., McKay, T. A., et al. 2008, MNRAS, 387, L28

Sakelliou, I., \& Ponman, T. J. 2004, MNRAS, 351, 1439

Sanderson, A. J. R., Ponman, T. J., \& O'Sullivan, E. 2006, MNRAS, 372, 1496

Sarazin, C. L. 1988, X-ray emission from clusters of galaxies, Cambridge Astrophys. Ser. (Cambridge: Cambridge University Press)

Snowden, S. L., Mushotzky, R. F., Kuntz, K. D., \& Davis, D. S. 2008, A\&A, 478,615

Stanek, R., Evrard, A. E., Böhringer, H., Schuecker, P., \& Nord, B. 2006, ApJ, 648,956

Tozzi, P., Rosati, P., Ettori, S., et al. 2003, ApJ, 593, 705

Watanabe, M., Yamashita, K., Furuzawa, A., et al. 1999, ApJ, 527, 80

Werner, N., Durret, F., Ohashi, T., Schindler, S., \& Wiersma, R. P. C. 2008, Space Sci. Rev., 134, 337

Wik, D. R., Sarazin, C. L., Ricker, P. M., \& Randall, S. W. 2008, ApJ, 680, 17 Wright, E. L. 2006, PASP, 118, 1711 